\title{
Kinematic and reduced-dynamic precise orbit determination of low earth orbiters
}

\author{
D. $\check{S ̆}$ ehla ${ }^{1}$ and M. Rothacher ${ }^{2}$ \\ ${ }^{1}$ Institut für Astronomische and Physikalische Geodäsie (IAPG), Technische Universität München, Arcisstrasse 21, D-80333 \\ München, Germany \\ ${ }^{2}$ Forschungseinrichtung Satellitengeodäsie (FESG), Technische Universität München, Arcisstrasse 21, D-80333 München, \\ Germany
}

\begin{abstract}
Various methods for kinematic and reduced-dynamic precise orbit determination (POD) of Low Earth Orbiters (LEO) were developed based on zero- and double-differencing of GPS carrier-phase measurements with and without ambiguity resolution. In this paper we present the following approaches in LEO precise orbit determination:

- zero-difference kinematic POD,

- zero-difference dynamic POD,

- double-difference kinematic POD with and without ambiguity resolution,

- double-difference dynamic POD with and without ambiguity resolution,

- combined GPS/SLR reduced-dynamic POD.
\end{abstract}

All developed POD approaches except the combination of GPS/SLR were tested using real CHAMP data (May 20-30, 2001) and independently validated with Satellite Laser Ranging (SLR) data over the same 11 days.

With SLR measurements, additional combinations are possible and in that case one can speak of combined kinematic or combined reduced-dynamic POD. First results of such a combined GPS/SLR POD will be presented, too.

This paper shows what LEO orbit accuracy may be achieved with GPS using different strategies including zerodifference and double-difference approaches. Kinematic versus dynamic orbit determination is presently an interesting issue that will also be discussed in this article.

Key words. POD, kinematic orbit, dynamic orbit, LEO, CHAMP, ambiguity resolution, GPS, SLR

\section{Introduction}

Today more and more low Earth orbiting satellites (LEOs) of new scientific missions are equipped with a GPS receiver

Correspondence to: D. Švehla (svehla@bv.tum.de) for precise orbit determination (POD). Although POD with GPS has been tested using data from various satellites (e.g. TOPEX/Poseidon, GPS/MET, etc.), there are still many open issues concerning the optimum way to determine LEO satellite orbits with GPS: on one hand the quality of spaceborne GPS receivers has considerably improved, and on the other hand much progress was achieved in the modeling aspects of POD.

Over the last year we developed various methods to compute LEO satellite orbits based on techniques ranging from reduced-dynamic to purely kinematic precise orbit determination. These algorithms allow to process GPS code and phase observations on the zero- or double-difference level. They have been thoroughly tested using simulated data and various analyses of real CHAMP data have been performed.

By making use of dynamical models (e.g. Earth's gravity field, tides, air-drag, solar radiation pressure) satellite orbits can be determined using different types of measurements, e.g. pseudo-range, carrier-phase (GPS), range (SLR) and doppler type of measurements (DORIS). In this case the quality of the dynamical models are crucial for the orbit obtained. On the other hand, the GPS technique, by tracking many satellites every epoch, allows a purely kinematic approach without making use of any dynamical model. Kinematic orbit determination is independent of the gravity field and of all the non-conservative forces acting on the satellites.

In the first part of the paper the observation equations and the treatment of the parameters in all methods will be presented and in the following, results for the CHAMP satellite, including the validation with SLR, will be given.

\section{LEO GPS observation equation}

The observation equation for LEO zero-difference POD using carrier-phase measurements for the frequency $i$ between LEO receiver and GPS satellite $s$ can be written as follows (in units of length):

$L_{L E O, i}^{s}=\rho_{\mathrm{LEO}}^{s}+c\left(\delta t_{\mathrm{LEO}}+\delta t_{s y s, i}\right)-c\left(\delta t^{s}+\delta t^{s y s, i}\right)+$ 


$$
\begin{aligned}
& +\delta \rho_{i o n, i}+\delta \rho_{r e l}+\delta \rho_{m u l, i}+\delta \rho_{p c o, i}+\delta \rho_{p c v, i}+ \\
& +\lambda_{i} \cdot N_{L E O, i}^{s}+\epsilon_{i}
\end{aligned}
$$

$L_{L E O, i}^{s} \quad$ LEO zero-diff. phase measurements, $\rho_{\text {LEO }}^{S} \quad$ geometrical distance, $c \quad$ speed of light in vacuum,

$\delta t_{\text {LEO }}, \delta t^{s} \quad$ LEO and GPS satellite clock corrections,

$\delta t_{s y s, i}, \delta t^{s y s, i}$ LEO and GPS satellite system delays (cable, electronics),

$\delta \rho_{\text {ion }, i} \quad$ ionospheric delay,

$\delta \rho_{\text {rel }} \quad$ relativistic correction,

$\delta \rho_{m u l, i} \quad$ multipath,scattering, bending effects,

$\delta \rho_{p c o, i} \quad$ LEO phase center offset,

$\delta \rho_{p c v, i} \quad$ LEO phase center variations,

$\lambda_{i} \quad$ wavelength of the GPS signal $\left(L_{1}\right.$ or $\left.L_{2}\right)$,

$N_{L E O, i}^{S} \quad$ zero-difference phase ambiguity,

$\epsilon_{i} \quad$ phase noise $\left(L_{1}\right.$ or $\left.L_{2}\right)$

One can immediately recognize the well-known observation equation for a ground station with one exception: in the LEO case there is no tropospheric delay to be taken into account. In order to eliminate ionospheric delays, the ionosphere-free $\left(L_{3}\right)$ linear combination (LC) can be formed between the LEO phase measurements $L_{\mathrm{LEO}, 1}$ and $L_{\mathrm{LEO}, 2}$ on carrier frequencies $f_{1}$ and $f_{2}$, respectively:

$L_{\mathrm{LEO}, 3}^{s}=\frac{f_{1}^{2}}{f_{1}^{2}-f_{2}^{2}} L_{\mathrm{LEO}, 1}^{s}-\frac{f_{2}^{2}}{f_{1}^{2}-f_{2}^{2}} L_{\mathrm{LEO}, 2}^{s}$

In this case the LEO zero-difference observation equation can be written as follows

$$
\begin{aligned}
& L_{\mathrm{LEO}, 3}^{s}=\rho_{\mathrm{LEO}}^{s}+c \cdot \delta t_{\mathrm{LEO}, \mathrm{clk}, 3}-c \cdot \delta t^{s, c l k, 3}+ \\
& \quad+\delta \rho_{r e l}+\delta \rho_{m u l, 3}+\delta \rho_{p c o, 3}+\delta \rho_{p c v, 3}+ \\
& \quad+B_{\mathrm{LEO}, 3}^{s}+\epsilon_{3}
\end{aligned}
$$

where $\delta t_{\mathrm{LEO}, \mathrm{clk}, 3}$ denotes the ionosphere-free LEO clock parameter consisting of the real LEO clock value $\delta t_{\text {LEO }}$ and the system delays $\delta t_{s y s, 1}$ and $\delta t_{s y s, 2}$ on both frequencies:

$\delta t_{\mathrm{LEO}, \mathrm{clk}, 3}=\delta t_{\mathrm{LEO}}+\frac{f_{1}^{2}}{f_{1}^{2}-f_{2}^{2}} \delta t_{s y s, 1}-\frac{f_{2}^{2}}{f_{1}^{2}-f_{2}^{2}} \delta t_{s y s, 2}$.

In the same way the ionosphere-free GPS clock parameter can be defined as

$\delta t^{s, c l k, 3}=\delta t^{s}+\frac{f_{1}^{2}}{f_{1}^{2}-f_{2}^{2}} \delta t^{s y s, 1}-\frac{f_{2}^{2}}{f_{1}^{2}-f_{2}^{2}} \delta t^{s y s, 2}$.

$\delta \rho_{m u l, 3}, \delta \rho_{p c o, 3}$, and $\delta \rho_{p c v, 3}$ denote multipath effects, phasecenter offset and phase-center variations for the ionospherefree linear combination. The zero-difference ionosphere-free ambiguity (phase bias) is denoted by $B_{\mathrm{LEO}, 3}^{s}$.

The observation equation for the LEO zero-difference code measurements can be written in the same way with the exception that the LEO phase ambiguity parameter $B_{\mathrm{LEO}, 3}^{S}$ is not included. GPS satellite and LEO system delays are different for $P_{1}$ and $P_{2}$ code measurements. By convention, the ionosphere-free LC is said to have no differential code bias (DCB), i.e. system delays are included in the GPS satellite and the receiver clocks, respectively, see Schaer (1999).

The observation equation for POD based on double differences can be written by forming double-differences between the LEO and a ground station and between GPS satellites $k$ and $s$ :

$L_{\mathrm{grd}, \mathrm{LEO}, 3}^{s, k}=\left(L_{\mathrm{LEO}, 3}^{k}-L_{\mathrm{grd}, 3}^{k}\right)-\left(L_{\mathrm{LEO}, 3}^{s}-L_{\mathrm{grd}, 3}^{s}\right)$

In this way we can form baselines between all ground IGS points and the LEO. It is very important to note that, by using double-differences between LEO and ground station, the absolute tropospheric delay for the ground station can be estimated and isolated.

As soon as we involve the GPS ground network (e.g. the IGS network), the troposphere zenith delays and station coordinates have to be considered. In our POD approach weekly IGS solutions for station coordinates computed at the CODE Analysis Center and corresponding troposphere zenith delays and troposphere gradients are introduced and kept fixed, see IGS (2000).

\section{LEO kinematic precise orbit determination}

In this section kinematic POD will be considered from the point of view of zero- and double-difference carrier-phase and code GPS measurements.

\subsection{Zero-difference kinematic POD}

In the zero-diff. kinematic POD for each epoch three LEO coordinates have to be estimated together with one LEO clock parameter. Zero-diff. ambiguities are the only parameters in the adjustment procedure, that are not epochspecific. Figure 1 shows the normal equation matrix for zero-diff. kinematic POD over eleven epochs. On the main diagonal we can easily recognize $3 \times 3$ blocks of epochwise kinematic LEO coordinates, eleven epoch-wise LEO clock parameters and, in the lower right corner, 6 zerodiff. ionosphere-free ambiguities. We easily see the correlations between zero-difference ambiguities and epoch-wise parameters.

All zero-diff. approaches rely on the availability of highly accurate GPS satellite orbits and clocks, see IGS (2000). They should be provided with the same sampling as used for the LEO kinematic POD. It is very important that GPS satellite orbits and clocks are consistent with each other because of the high correlations. If highly accurate GPS satellite orbits and clocks are available, this method is very simple and reliable because it does not involve the huge load of processing the ground IGS network.

An alternative zero-diff. approach based on forming differences between phase observations of consecutive epochs and avoiding zero-difference ambiguity parameters, may be found in Bock et al. (2001). 


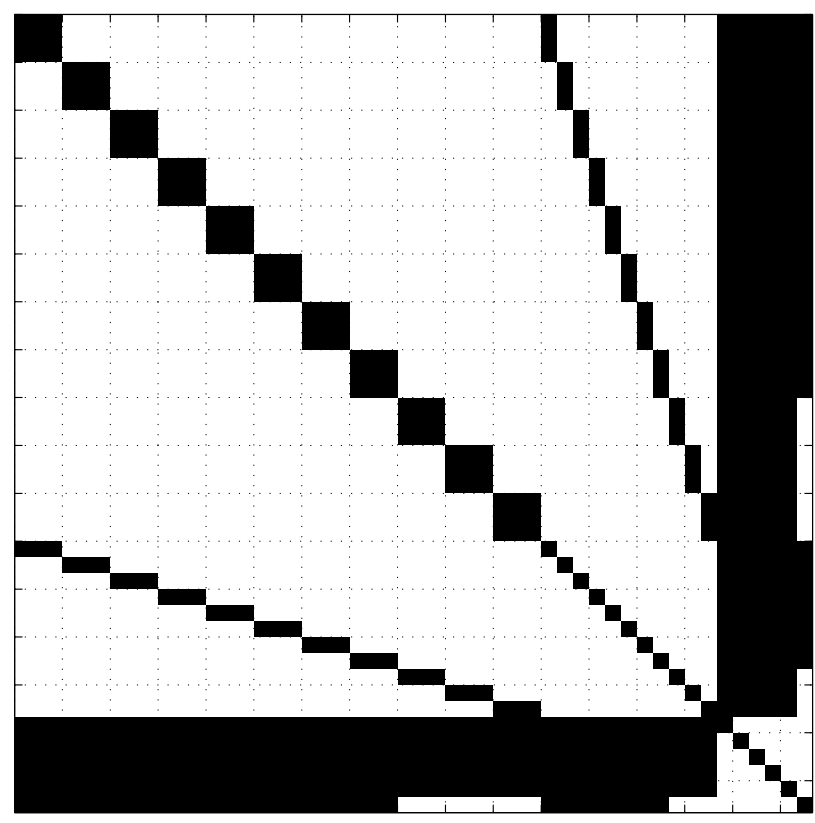

Fig. 1. Normal equation matrix for zero-diff. kinematic POD (11 epochs). On the main diagonal: 3 by 3 blocks of epoch-wise kinematic coordinates, 11 epoch-wise LEO clocks parameters and in the lower right corner 6 zero-difference ambiguity parameters.

\subsection{Double-difference kinematic POD}

In contrast to the zero-difference kinematic POD approach, the double-difference approach requires the simultaneous processing of the GPS ground network and the LEO GPS measurements. All possible baselines between LEO and ground IGS network are formed and processed together. For each epoch three kinematic LEO coordinates are estimated together with the double-difference ambiguity parameters. By forming double-differences, all GPS satellite clocks are eliminated and there is no need for highly accurate GPS satellite clocks computed from the GPS ground network.

The disadvantage of the double-difference kinematic approach is the huge number of observations and ambiguity parameters stemming from the IGS ground network. The noise of the double-difference observable is twice as high as that of the zero-difference observable, but we get rid of all clock parameters and, what is the most important, ambiguity resolution can be performed using double-differences.

An alternative double-diff. approach based on Kalman filtering with a high efficiency may be found in Colombo et al. (2002).

\section{LEO dynamic Precise Orbit Determination}

Dynamic POD approaches rely on the dynamical models, i.e. on the solution of the LEO equation of motion given by

$\ddot{\mathbf{r}}=-G M \frac{\mathbf{r}}{r^{3}}+\mathbf{f}_{\mathbf{1}}\left(t, \mathbf{r}, \dot{\mathbf{r}}, q_{1}, q_{2}, \ldots, q_{d}\right)=\mathbf{f}_{\mathbf{0}}+\mathbf{f}_{\mathbf{1}}=\mathbf{f}$ r LEO geocentric position (J2000),

$t$ dynamic time (TT),

$G M$ gravity constant times mass of the Earth,

$\mathbf{f}_{0}$ acceleration due to the central gravity term,

$\mathbf{f}_{1}$ perturbing accelerations acting on the LEO,

$q_{1}, \ldots, q_{d}$ LEO dynamical orbit parameters,

f total LEO acceleration

where the LEO initial conditions (position $\mathbf{r}$ and velocity $\dot{\mathbf{r}}$ ) in the inertial system at the reference epoch $t_{0}$ can be represented using the well-known Keplerian elements (semimajor axis $a$, eccentricity $e$, inclination $i$, ascending node $\Omega$, argument of perigee $\omega$ and perigee passing time $t_{p}$ ) at $t_{0}$ :

$\mathbf{r}\left(t_{0}\right)=\mathbf{r}\left(a, e, i, \Omega, \omega, t_{p} ; t_{0}\right)$

$\dot{\mathbf{r}}\left(t_{0}\right)=\dot{\mathbf{r}}\left(a, e, i, \Omega, \omega, t_{p} ; t_{0}\right)$.

The perturbing accelerations that act on the LEO are due to the higher order terms of the gravity field (e.g. given by EIGEN1S, see Reigber et al., 2002), air-drag (e.g. from the model MSISE-90, see Hedin, 1991; Doornbos et al., 2002), solar radiation pressure, contributions of direct tidal effects from all third bodies as the Sun, the Moon and the planets, as well as indirect tidal effects (solid Earth tides, ocean tides) and general relativistic effects. Earth albedo could also be included, although its modeling is relatively complicated due to the fact that information on cloud coverage and ocean surfaces has to be taken into account. The same equation of motion is valid for GPS satellites, but the effects of the gravity field of the Earth are much weaker and air-drag is nonpresent.

Dynamic orbit determination can be considered as an orbit improvement procedure, where the orbit $\mathbf{r}(t)$ is developed into a Taylor series with respect to the unknown orbit parameters $p_{i}(i=1, \ldots, n)$ about the a priori dynamic orbit $\mathbf{r}_{0}(t)$, which is based on the a priori values $p_{i 0}$ of the orbital parameters:

$\mathbf{r}(t)=\mathbf{r}_{0}(t)+\sum_{i=1}^{n} \frac{\partial \mathbf{r}_{0}(t)}{\partial p_{i}}\left(p_{i}-p_{i 0}\right)$

The parameters $p_{i}$ include the initial state vector (position and velocity) and all the dynamical parameters $q_{1}, \ldots, q_{d}$ that define the satellite force model. The partial derivatives $\frac{\partial \mathbf{r}_{0}}{\partial p_{i}}$ are computed from the so-called variational equations (obtained as the partial derivative of the equation of motion, Eq. 7) with respect to the parameters $p_{i}$. The LEO equation of motion as well as all variational equations are usually obtained by a numerical integration.

The use of so-called pseudo-stochastic pulses or small changes in the velocity at user-determined epochs was developed for GPS orbits by the CODE Analysis Center Team, see Rothacher and Mervart (1996), Beutler, et al. (1994). The estimation of such pulses can easily be implemented into the orbit determination procedure due to the nice feature that the partials for such pulses can be computed as a linear combination of the partials with respect to the initial conditions. 
Frequently setting up stochastic pulses may be considered equivalent to modeling air-drag and other forces with many parameters. The pseudo-stochastic pulses allow a better fit of the orbit to the measurements and are set up every 15 or even 9 minutes in our case.

In summary, the LEO dynamic orbit is parametrized in our case by

- 6 initial conditions (Keplerian elements),

- 9 solar radiation pressure parameters,

- 1 air-drag scaling factor,

- pseudo-stochastic pulses every 9 or $15 \mathrm{~min}$ in alongtrack, cross-track and radial direction.

\subsection{Zero-difference dynamic POD}

The only difference between dynamic and kinematic zerodifference POD is, that kinematic coordinates for each epoch are replaced by the orbital parameters mentioned above. This means that the dynamical orbit parameters have to be estimated together with zero-diff. ambiguities and epoch-wise LEO clocks. In both cases high accuracy GPS clocks and GPS orbits have to be available. By replacing the kinematic by a dynamic orbit modelling the number of parameters is considerably reduced and LEO satellite clock corrections are the only remaining epoch-wise parameters. Later in this paper we will show with real CHAMP data, that both approaches provide orbits of similar quality. The quality of the GPS satellite clocks used and the number of GPS satellites tracked by the spaceborne LEO receiver are the two main factors that influence the quality of the zero-difference kinematic POD, whereas the quality of the dynamical models and parameterization and GPS satellite clocks primarily determine the accuracy of the dynamic zero-difference POD.

\subsection{Double-difference dynamic POD}

In the case of double-difference dynamic POD the only parameters to be estimated are the double-difference ambiguities and the orbital parameters. Highly accurate GPS satellite clocks are not required for this method.

\section{Parameter treatment in kinematic and dynamic POD}

Table 1 shows the parameter statistics for zero- and doubledifference kinematic and dynamic POD with real CHAMP data over one day. We immediately notice the huge number of phase observations stemming from the about 100 IGS ground stations we selected. This together with the fast changing geometry is also the reason why a huge number of double-difference ambiguities are involved. Compared to dynamic parameterization, kinematic POD has much more epoch-wise parameters. Table 2 shows the treatment of parameters while forming the normal equation system. In order to speed up computation, epoch-wise parameters (LEO clocks and kinematic positions) are always pre-eliminated
Table 1. Parameter and observation statistics for zero-difference (ZD) and double-difference (DD) dynamic and kinematic POD (for CHAMP, day 148 in 2001)

\begin{tabular}{lcccc}
\hline Solution & ZD Dyn & ZD Kin & DD Dyn & DD Kin \\
\hline Ambigiguitis & 450 & 450 & 13200 & 13200 \\
Orbit Parameters & 300 & - & 300 & - \\
Kinematic Coordinates & - & 8640 & - & 8640 \\
LEO Clocks & 2880 & 2880 & - & - \\
\hline Total Number of Param. & 3630 & 11700 & 13500 & 21840 \\
\hline Number of Observations & 18400 & 18400 & 340000 & 340000 \\
\hline
\end{tabular}

Table 2. Treatment of parameters in the zero- (ZD) and doubledifference (DD) dynamic and kinematic POD (CHAMP, day 148 in 2001)

\begin{tabular}{lcccc}
\hline Solution & ZD Dyn & ZD Kin & DD Dyn & DD Kin \\
\hline Ambiguities & pre-elim. & estim. & pre-elim. & pre-elim. \\
Orbit Parameters & estim & - & estim. & - \\
Kinematic Coord. & - & pre-elim. & - & estim. \\
LEO Clocks & pre-elim. & pre-elim. & - & - \\
Time & $24 \mathrm{~h}$ & $24 \mathrm{~h}$ & $24 \mathrm{~h}$ & $8 \mathrm{~h}$ \\
CPU & $10 \mathrm{~min}$ & $10 \mathrm{~min}$ & $90 \mathrm{~min}$ & $60 \mathrm{~min}$ \\
\hline
\end{tabular}

epoch-by-epoch. At the end, only the normal equation matrix consisting of parameters, that are not epoch-specific, is inverted and in sequel, by back substitution, epoch-wise parameters are obtained epoch-by-epoch. In the doubledifference kinematic case we have to pre-eliminate doubledifference ambiguities because of computer memory consideration. We also see that the zero-diff. approaches are much faster than double-differencing and usually (with data preprocessing and the estimation of an a priori orbit) they require less that 10 minutes on a standard PC $(800 \mathrm{MHz})$.

\section{Ambiguity resolution in kinematic and dynamic POD}

Only the double-difference approaches allow for ambiguity resolution and this is why, in principle, the highest accuracy should be achievable with these methods. In this section, only the results of ambiguity resolution will be presented. Details on the theoretical background can be found in Švehla and Rothacher (2002a) and in Švehla and Rothacher (2002b). Our ambiguity resolution approach is based on the fixing of wide-lane ambiguities using the Melbourne-Wübbena linear combination and subsequent fixing of narrow-lane ambiguities using the ionosphere-free linear combination of phase measurements only. In this way, the ionospheric delays that show up in the classical wide-laning (phase only) technique are eliminated. The main reason why this method works very well is, that CHAMP code measurements are very accurate. On the other hand, the QIF (quasi-ionosphere-free) ambigu- 


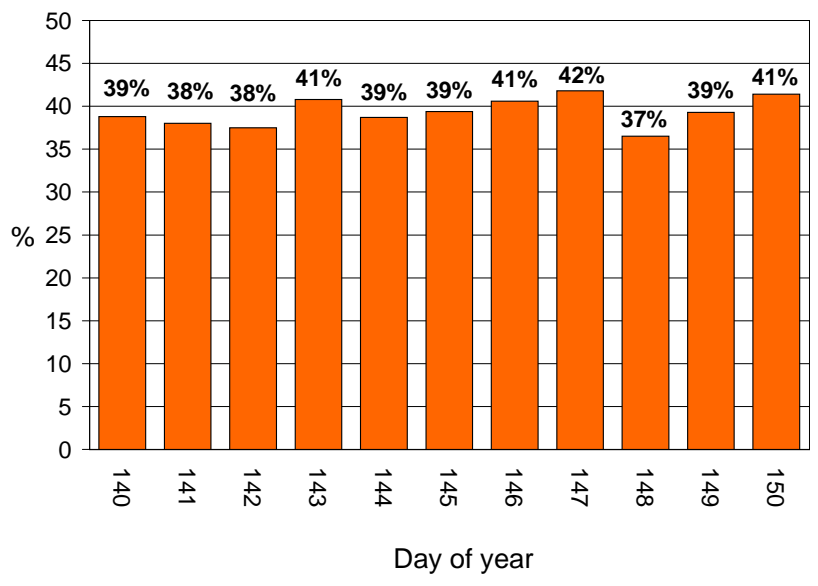

Fig. 2. Percentage of resolved wide-lane ambiguities over 11 days using the Melbourne-Wübbena LC.

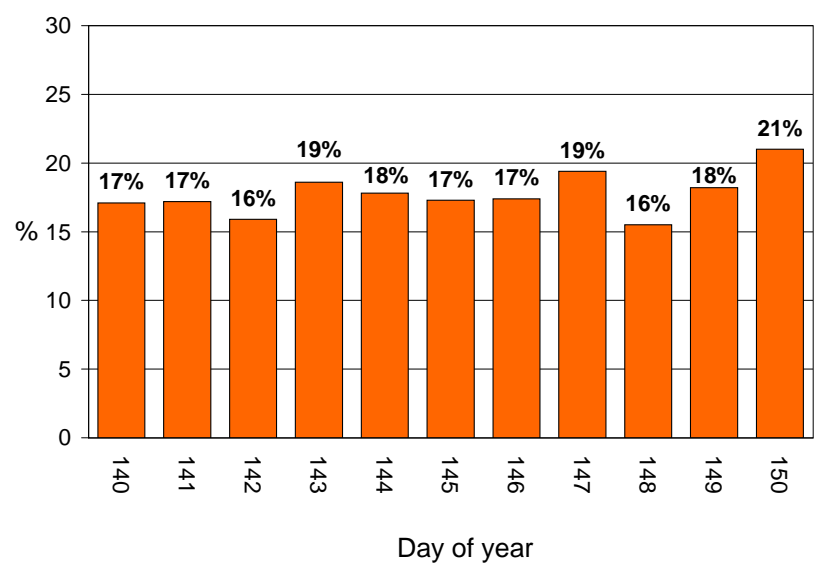

Fig. 3. Percentage of resolved narrow-lane ambiguities over 11 days.

ity resolution strategy (see, Mervart, 1995) still does not provide satisfactory results due to the difficulties in the modeling of the fast changing ionosphere environment for CHAMP. Figure 2 shows the percentage of the resolved wide-lane ambiguities over 11 days and Fig. 3 the percentage of resolved narrow-lane ambiguities over the same test period. Although the percentage of resolved ambiguities might seem small, it will be shown that it has a considerable impact on the kinematic orbit quality.

\section{SLR validation}

All developed approaches described in this paper were tested with real CHAMP data over a period of 11 days (days 140$150,2001)$ and validated with SLR measurements. The SLR residuals were computed as the difference between the SLR measurements (corrected for the tropospheric delay with the Marini-Murray model) minus the distance between the SLR station and the GPS-derived orbit position. Altogether 2007

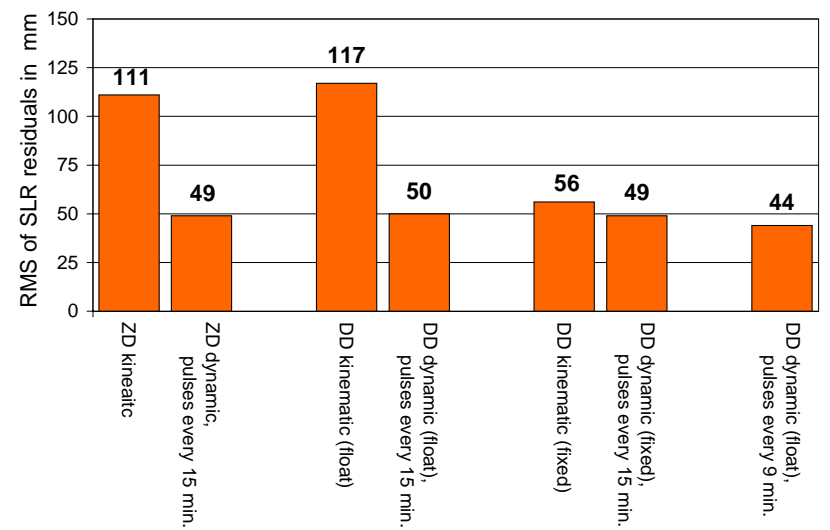

Fig. 4. RMS of SLR residuals for all POD strategies (CHAMP, days 140-150, year 2001).

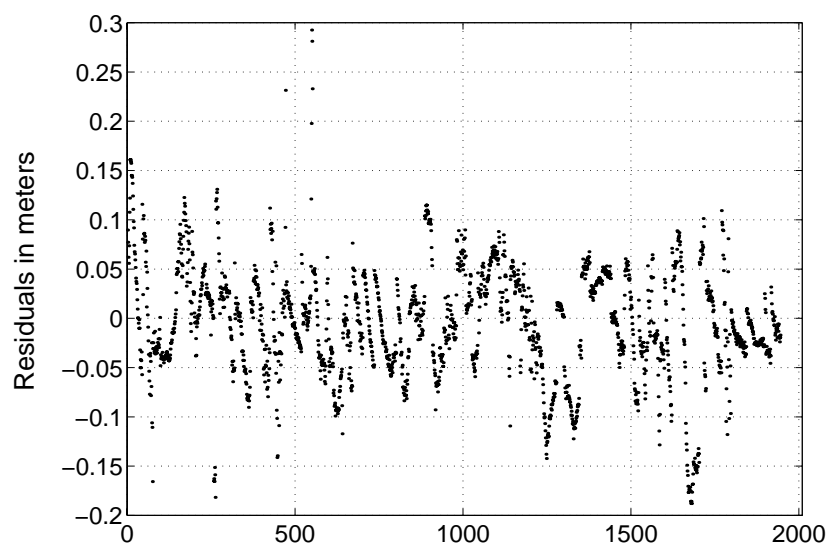

Fig. 5. SLR residuals for kinematic orbit with resolved ambiguities $(\mathrm{RMS}=56.4 \mathrm{~mm})$.

SLR residuals were obtained in this way using 69 daily station files from 17 SLR stations. For the validation of dynamic orbits, the LEO positions were computed directly from the dynamic orbit representation for the epoch, when the SLR measurement took place. The offset between CHAMP center of mass and SLR retro-reflector was applied using attitude computed from Advanced Stellar Compass data. In the case of kinematic orbits, the only difference is, that the kinematic orbits are given with a sampling of $30 \mathrm{~s}$ and an interpolation procedure is required in order to obtain positions at the epochs of the SLR normal points. A linear interpolation was used to get positions along an a priori dynamic orbit. We noticed that the validation of kinematic orbits with SLR is more difficult and the necessary interpolation may easily increase the RMS by $1-2 \mathrm{~cm}$. Another alternative would be to form normal points exactly at the epochs, where kinematic positions are defined, but raw SLR data would have to be processed in this case and they are not easily available for all SLR stations (Gurtner, 2002).

Figure 4 shows that the highest accuracy of $4-6 \mathrm{~cm}$ was 


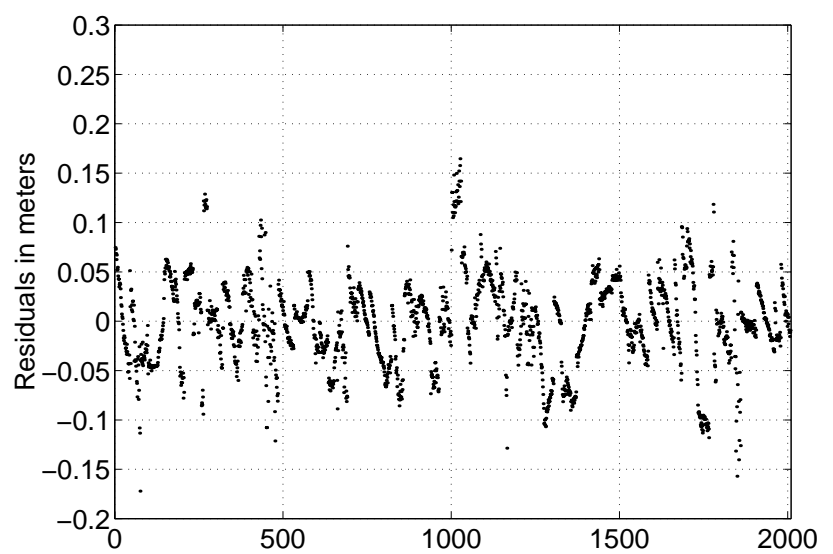

Fig. 6. SLR residuals for double-diff. dynamic orbit $(\mathrm{RMS}=44.3$ $\mathrm{mm}$ ), pseudo-stochastic pulses each $9 \mathrm{~min}$.

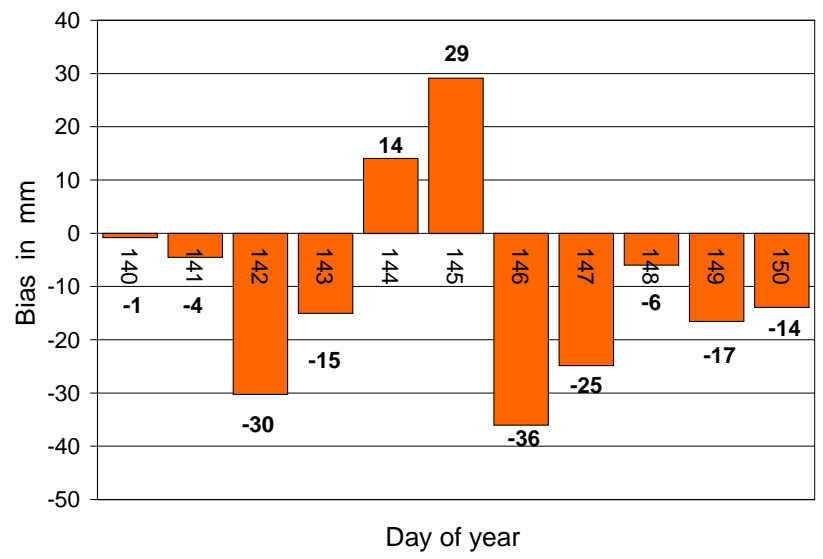

Fig. 7. Daily SLR range bias for zero-diff. dynamic orbit, mean SLR bias of $-11.1 \pm 19 \mathrm{~mm}$ over all SLR residuals.

achieved with double-difference dynamic orbits and doubledifference kinematic orbits with ambiguity resolution. The estimation of stochastic pulses every $9 \mathrm{~min}$ improves the orbit quality compared to the solution with 15 -min pulses by about $2 \mathrm{~cm}$ RMS-wise. The impact of ambiguity resolution on kinematic orbits is very remarkable, whereas for dynamic orbits it is relatively small and can be explained by the much smaller number of parameters for the reduced-dynamic orbit representation, see Fig. 4. It it very important to mention that in all kinematic and dynamic approaches no significant bias could be detected in the SLR residuals. For example, for double-difference dynamic orbits the bias is $-1.3 \pm 16 \mathrm{~mm}$, whereas for kinematic orbits it amounts to $-5.0 \pm 25 \mathrm{~mm}$ over 11 days, see Fig. 8 and Fig. 9. The zero-difference orbits show a similar behaviour with a bias of $-11.1 \pm 19 \mathrm{~mm}$ in the dynamic case, see Fig. 7.

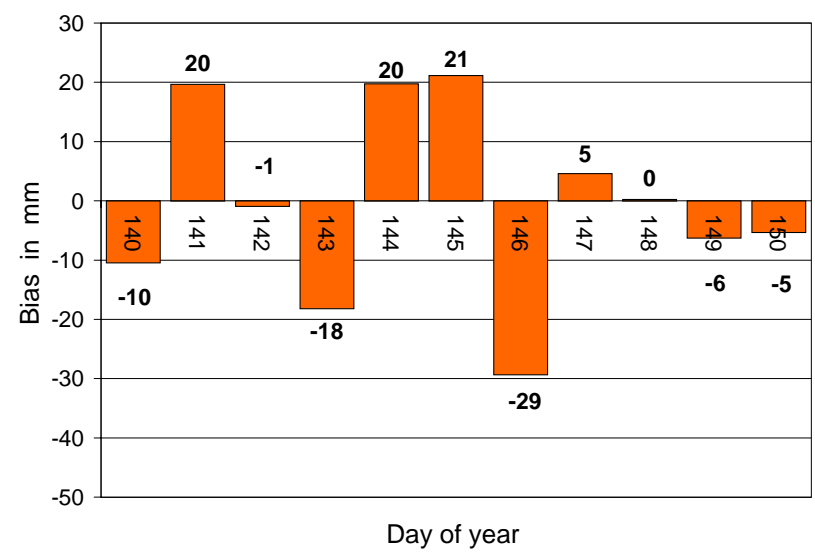

Fig. 8. Daily SLR range bias for double-diff. dynamic orbit, mean SLR bias of $-1.3 \pm 16 \mathrm{~mm}$ over all SLR residuals.

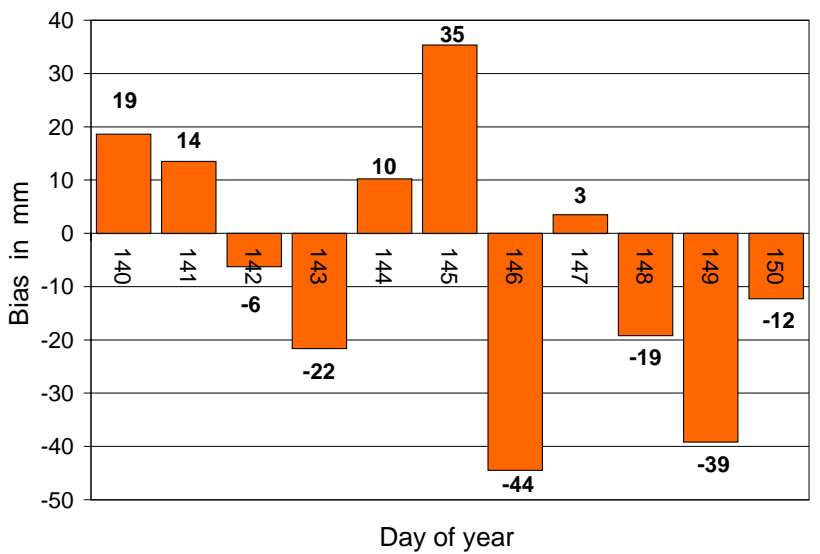

Fig. 9. Daily SLR range bias for double-diff. kinematic orbit, mean SLR bias of $-5.0 \pm 25 \mathrm{~mm}$ over all SLR residuals.

\section{Combined GPS/SLR Precise Orbit Determination}

Figure 10 shows the combined GPS/SLR dynamic POD orbit using SLR and zero-diff. phase measurements for day 144 in 2001 compared to the dynamic orbit estimated using the GPS measurements only. Troposphere effects were modeled using the Marini-Murray model and standard corrections like relativity, ocean-loading, station velocities were applied. Since GPS satellite orbits and coordinates of the ground GPS points were referring to ITRF97 in 2001, all SLR station coordinates given in ITRF2000 were transformed to ITRF97 and the combined LEO POD was done in this system. The computation was performed using 9 SLR stations.

The comparison between the combined GPS/SLR dynamic orbit and the GPS-only solution reveals that SLR measurements change the orbit by $1-2 \mathrm{~cm}$. This value, of course, heavily depends on the relative weighting between GPS phase and SLR measurements. Because it is very difficult to validate this combined orbit, the importance of the SLR data mainly resides in the fact that systematic biases in the satellite orbit model, the satellite attitude, GPS antenna phase cen- 

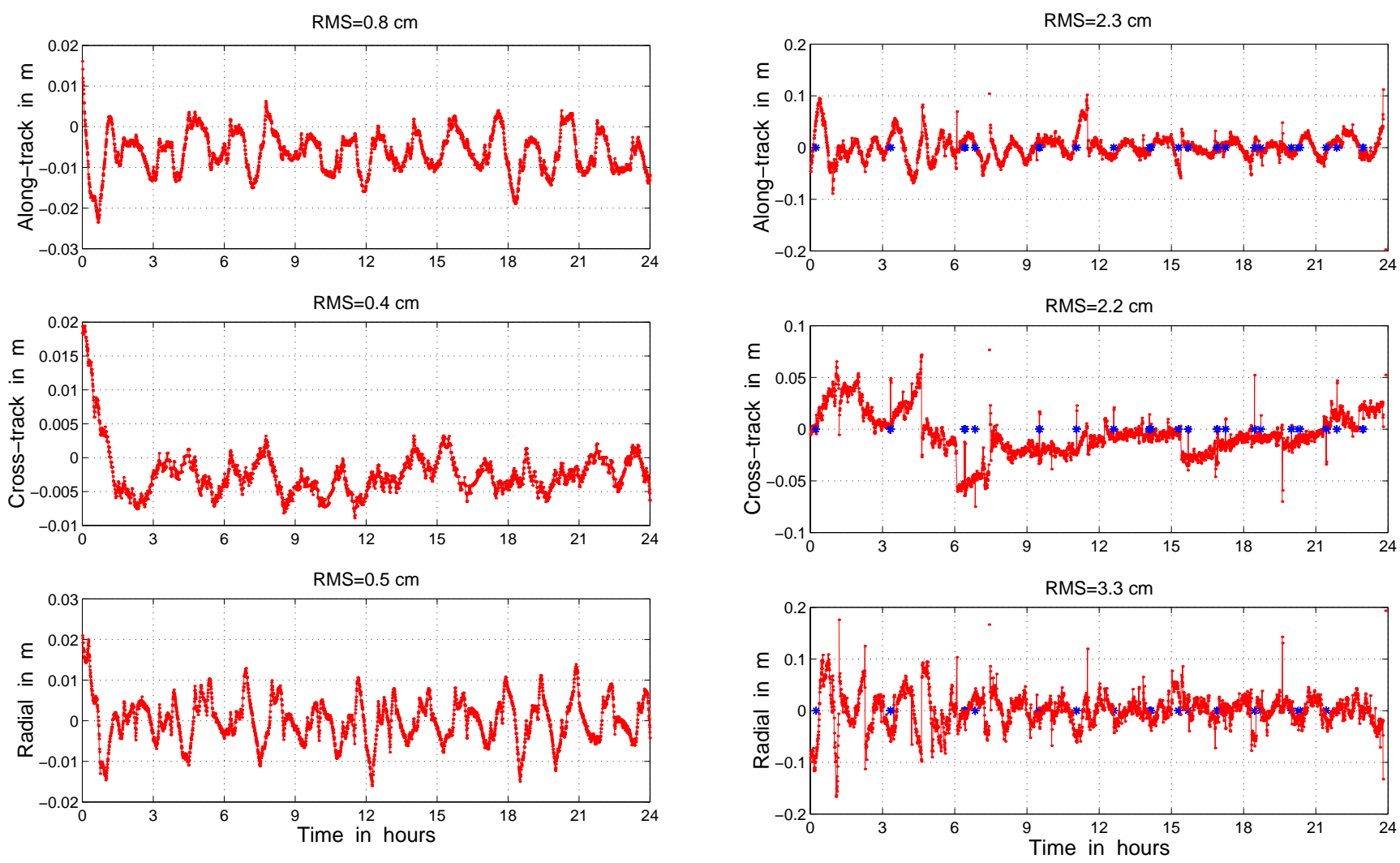

Fig. 10. Combined GPS/SLR dynamic zero-diff. POD compared to GPS zero-diff. dynamic POD, day 144/2001.

ter variations, etc. might be detected. Anyway, more detailed study should be performed with more recent CHAMP data (see next section) and the weighting of the SLR measurements.

In future, the combination of GPS and SLR data in the case of kinematic POD will be a very interesting topic to study, especially when using more recent CHAMP data. In this case SLR normal points should be formed at the GPS measurement epochs.

\section{First results with more recent CHAMP data}

In the year 2002, the CHAMP GPS receiver software was changed to enable the tracking of up to 10 GPS satellites at the same time. In order to assess the accuracy of kinematic and dynamic POD under these improved tracking conditions, we computed zero-difference kinematic and dynamic orbits for day 202/2002. Figure 11 compares these two orbit types and we see that they agree on the level of $2-3 \mathrm{~cm}$. Such a good agreement between kinematic and dynamic orbits clearly shows the impact of the increased number of GPS satellites in the more recent CHAMP data. The stars in the figure indicate epochs with missing attitude information (nominal attitude was used in this case) and jumps may be seen in the difference between the two orbit types at these epoch increasing the RMS difference. The assessment of

Fig. 11. Zero-diff. kinematic orbit compared to dynamic orbit, day 202/2002, starts are epochs with missing attitude information.

these kinematic and dynamic orbits with SLR measurements results in an RMS of $2.1 \mathrm{~cm}$ and $2.3 \mathrm{~cm}$, respectively.

\section{Kinematic and dynamic velocities}

Kinematic velocities of CHAMP are very interesting from the point of view of the energy conservation approach in gravity field recovery, see e.g. Gerlach et al. (2002).

Most simply, kinematic velocities can be obtained by forming time differences of kinematic positions, whereas dynamic velocities are directly obtained when solving the equations of motion by numerical integration. In order to compute unbiased kinematic velocities by forming time differences, the LEO positions should be given with a high sampling rate.

Let us first compare CHAMP dynamic velocities between several POD centers included in the CHAMP Orbit Comparison Campaign (see, Boomkamp, 2002a,b), namely between the Center for Space Research (CSR), Austin Texas, the GeoForschungsZentrum (GFZ), Potsdam, Germany, and the Technische Universität München (TUM), i.e. our dynamic velocities (see Figs. 12, 13 and 14). Just by comparison of the best CHAMP dynamic orbits, we can immediately draw the conclusion that CHAMP dynamic velocities with a precision below $0.1 \mathrm{~mm} / \mathrm{s}$ can be computed.

Kinematic velocities can be computed in several ways. One possibility is to fit a high-order polynomial to the kine- 

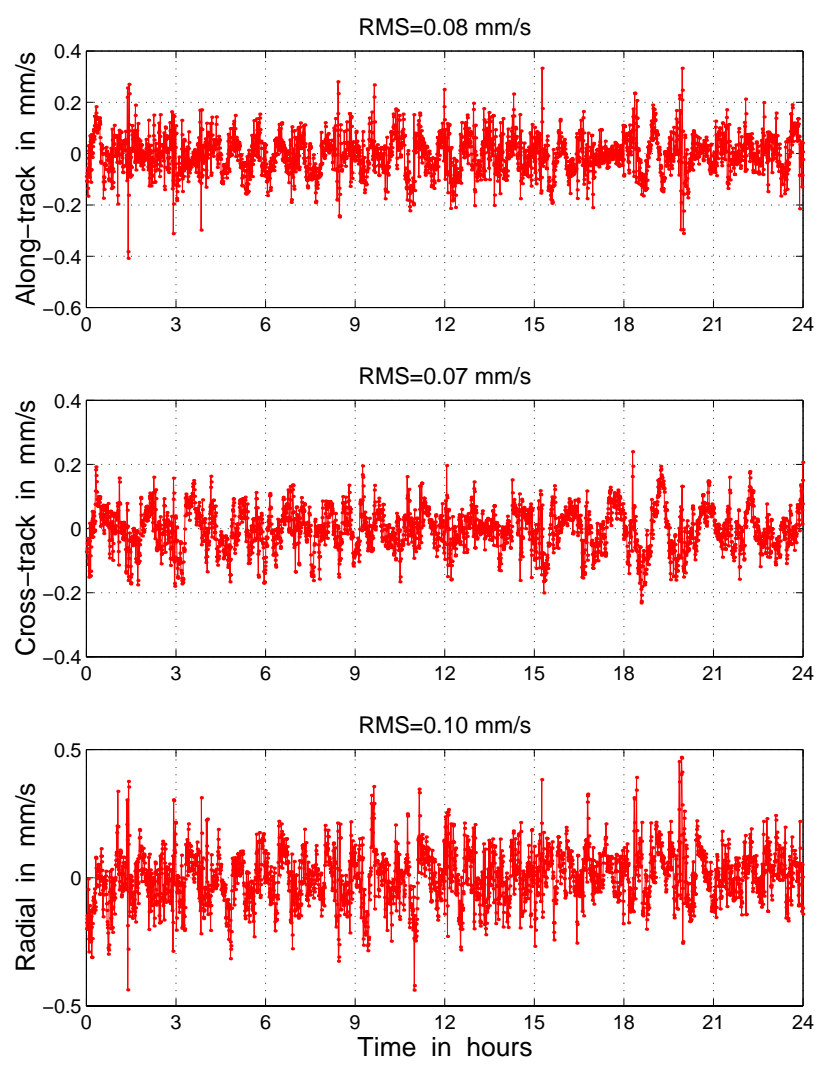

Fig. 12. Comparison of CHAMP dynamic velocities from CSR and TUM, day 148/2001.

matic positions and to compute the first derivative of such a polynomial. In this way a high-degree polynomial will fit kinematic positions over a given time span and at the same time smooth the kinematic positions. The second derivative of such a polynomial will directly lead to kinematic accelerations. In order to optimize this procedure, an a priori dynamic orbit can be used and the high-order polynomial can be fitted to the differences between kinematic orbit and dynamic a priori orbit. In this way, the kinematic velocity will be given as the sum of the dynamic velocity derived from the a priori dynamic orbit and the first derivative of the polynomial used. The main drawback of this method is that, depending on the polynomial degree used for smoothing, systematic effects at different frequencies may show up in the derived velocities. One alternative to this method is to completely avoid the use of any polynomial and any smoothing in this procedure and just use plain differencing in time. The only disadvantage is a somewhat increased noise level.

Figure 15 shows such non-smoothed kinematic CHAMP velocities compared to the CHAMP dynamic velocities. Our latest CHAMP zero-difference kinematic orbit and zerodiff. dynamic orbit were used in this computation. Figures 13 and 15 clearly show that the accuracy of present CHAMP kinematic velocities (without smoothing) is worse than that of dynamic velocities. The noise level is somewhat higher in the kinematic case, especially in the radial component, but by
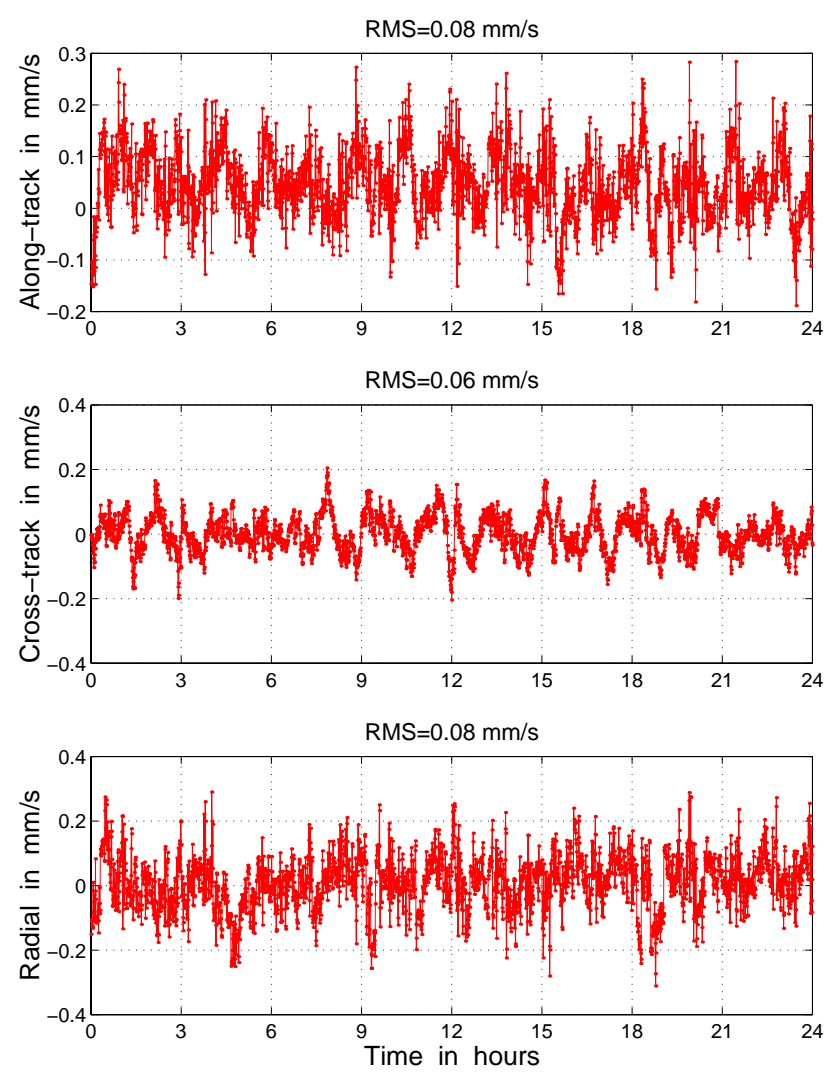

Fig. 13. Comparison of CHAMP dynamic velocities from GFZ and TUM, day 148/2001.

making use of var/covariance information for the kinematic positions, kinematic velocities can be reasonably weighted in the gravity field recovery.

\section{Conclusion}

Methods for kinematic and dynamic precise orbit determination (POD) of low Earth orbiters (LEO) were developed based on zero- and double-difference algorithms including ambiguity resolution:

- Zero-difference kinematic POD,

- Zero-difference dynamic POD,

- Double-difference kinematic POD (ambiguity-float),

- Double-difference dynamic POD (ambiguity-float),

- Double-difference kinematic POD (ambiguity-fixed),

- Double-difference dynamic POD (ambiguity-fixed),

- Combined GPS/SLR dynamic POD.

All POD approaches but the last one mentioned were presented and tested using 11 days of real CHAMP data (20 to 30 May 2001) and validated independently with Satellite Laser Ranging (SLR) measurements. This validation with SLR showed that double-diff. dynamic and doubledifference kinematic approach with ambiguity resolution are the two approaches providing the highest accuracy. 


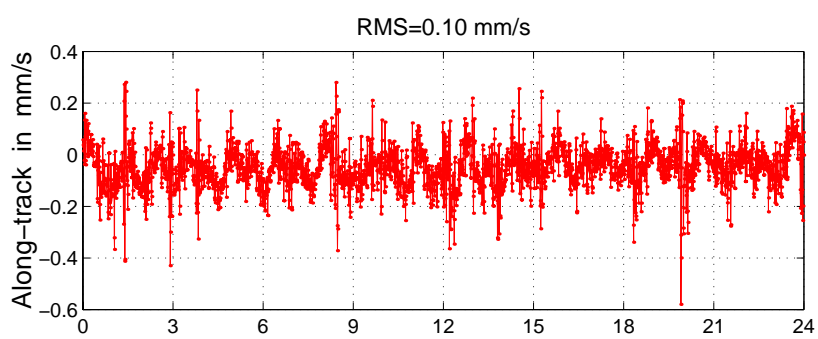

$\mathrm{RMS}=0.08 \mathrm{~mm} / \mathrm{s}$

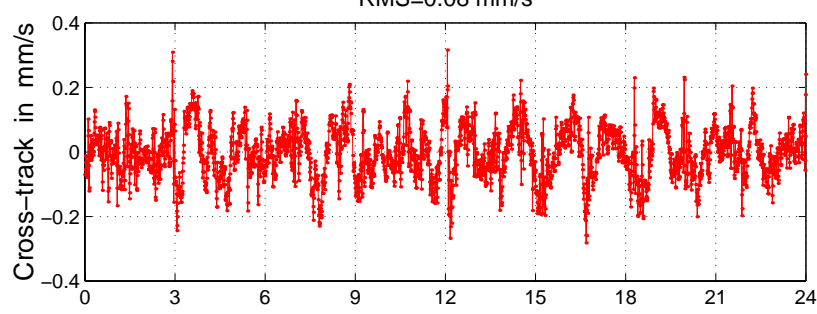

$\mathrm{RMS}=0.10 \mathrm{~mm} / \mathrm{s}$

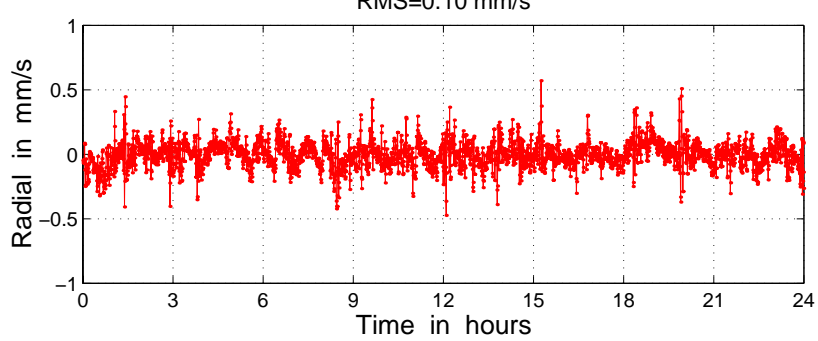

Fig. 14. Comparison of CHAMP dynamic velocities from GFZ and CSR, day $148 / 2001$.

The accuracy of the double-difference dynamic orbits is at a level of $4-5 \mathrm{~cm}(44.3 \mathrm{~mm})$, whereas the kinematic ambiguity-fixed orbits have a quality of about 5-6 cm $(56.4 \mathrm{~mm})$. Kinematic orbits of such a quality are extremely valuable to assess dynamical orbit models and parameterizations and may serve as input for gravity field recovery approaches. No significant SLR bias could be detected in the two orbit types. It is important to note that kinematic orbit positions should always be used together with the corresponding variancecovariance information. In addition, first results of a combined GPS/SLR POD were presented.

First results with more recent CHAMP GPS data (July 2002) shows that the number of GPS satellites tracked by the spaceborne GPS receiver plays a major role in kinematic POD. The comparison of kinematic orbits from July 2002, when up to 10 GPS satellites were tracked by the CHAMP GPS receiver, with kinematic orbits computed for the period of 11 days in the year 2001, where only up to 8 GPS satellites were tracked simultaneously, shows an increase in the accuracy by a factor of about two.

Acknowledgements. We are grateful to GeoForschungsZentrum Potsdam for providing GPS measurements from CHAMP satellite and German Academic Exchange Service for financial support through DAAD Scholarship.
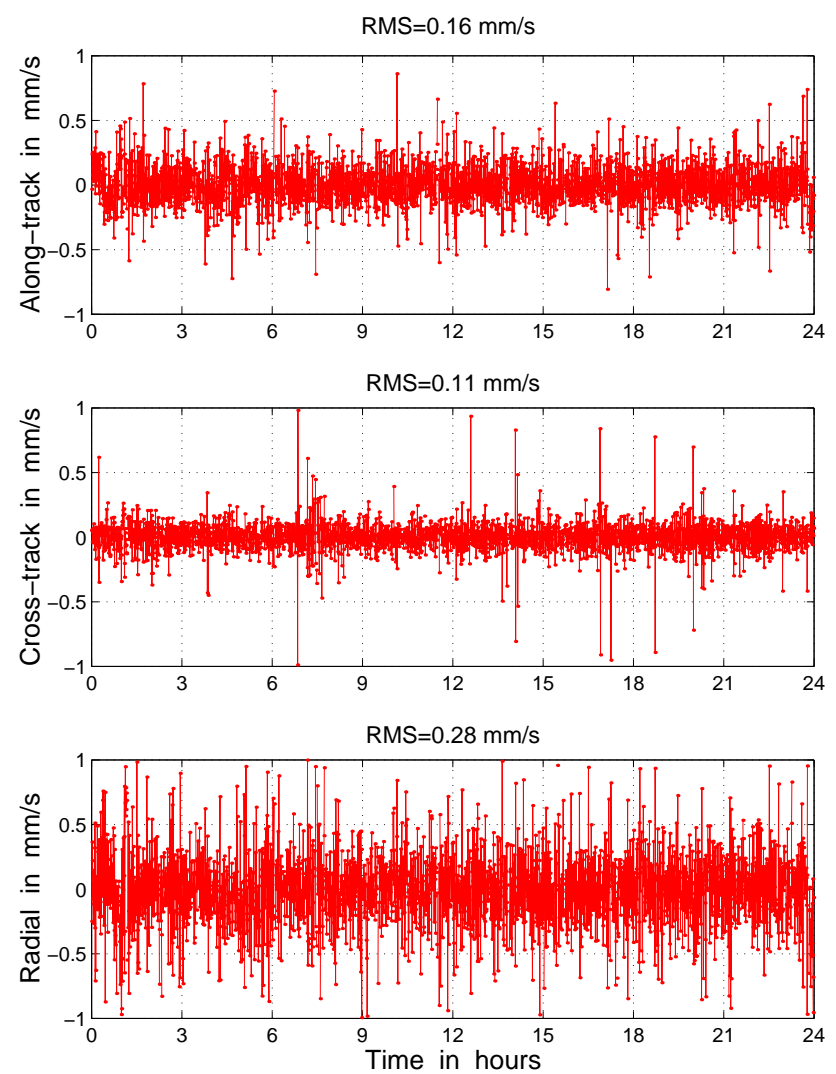

Fig. 15. Comparison of CHAMP kinematic (without smoothing) and dynamic velocities from TUM, day 202/2002

\section{References}

Beutler G., Brockmann E., Gurtner W., Hugentobler U., Mervart L., and Rothacher M.: Extended Orbit Modeling Techniques at the CODE Processing Center of the International GPS Service for Geodynamics (IGS): Theory and Initial Results, Manuscripta Geodaetica, 19, 367-386, 1994.

Bock, H., Hugentobler, U., and Beutler, G.: Kinematic Orbit determination for Low Earth Orbiters (LEOs), IAG 2001 Scientific Assembly, Budapest, Vistas for Geodesy in the New Millenium, (Eds) Adam, J. and Schwarz, K. P., Springer IAG, 125, 322-328, 2001.

Boomkamp, H.: IGS LEO Pilot Project associate Analysis Centre coordination at ESOC, Paper presented at 1st CHAMP Science Meeting, Potsdam, Germany, in print, 2002.

Boomkamp, H.: CHAMP Orbit Comparison Campaign, http://nng. esoc.esa.de/gps/campaign.html, 2002.

Colombo, O. L., Rowlands, D. D., Chin, D., and Poulouse, S.: Testing a GPS technique for precise Low Earth Orbit determination, Paper presented at European Geophysical Society, XXVII General Assembly, Nice, France, 21-26 April 2002.

Doornbos, E., Scharroo, R., Klinkrad H., Zandbergen R, and Fritsche B.: Improved modelling of surface forces in the orbit determination of ERS and Envisat, Canadian Journal of Remote Sensing, 28(4), 534-543, 2002.

Gerlach C., Sneeuw, N., Visser P., and Švehla, D.: CHAMP Gravity Field Recovery using the Energy Balance Approach, Paper presented at European Geophysical Society, XXVII General As- 
sembly, Nice, France, 21-26 April 2002, Adv. Geosciences, in press, 2003.

Gurtner, W.: Personal comunications, 2002.

Hedin, A. E.: Extension of the MSIS Thermosphere Model into the Middle and Lower Atmosphere, J. Geophys. Res., 96, 1159, 1991.

IGS: 2000 IGS Technical Reports, (Eds) IGS Central Bureau, JPL Pasadena, California, 2000.

Mervart, L.: Ambiguity Resolution Techniques in Geodetic and Geodynamic Application of the Global Positioning System, Vol. 53 of Geodätisch-geophys, Arbeiten in der Schweiz, Schweizerische Geodätische Kommission, Switzerland, 1995.

Reigber, Ch., Balmino, G., Schwintzer, P., Biancale, R., Bode, A., Lemoine, J.-M., Koenig, R., Loyer, S., Neumayer, H., Marty, J.-C., Barthelmes, F., Perosanz, F., and Zhu, S. Y.: A highquality global gravity field model from CHAMP GPS tracking data and accelerometry (EIGEN-1S), Geophys. Res. Lett.,
29(14), 10.1029/2002GL015064, 2002.

Rothacher, M. and Mervart, L.: (Eds), The Bernese GPS Software Version 4.0. Astronomical Institute, University of Berne, 1996.

Schaer, S.: Mapping and Predicting the Earth's Ionosphere Using the Global Positioning System, Vol. 59 of Geodätisch-geophys, Arbeiten in der Schweiz, Schweizerische Geodätische Kommission, Switzerland, 1999.

Švehla D. and Rothacher M.: Kinematic Orbit Determination of LEOs Based on Zero- or Double-Difference Algorithms Using Simulated and Real SST Data, IAG 2001 Scientific Assembly, Budapest, Vistas for Geodesy in the New Millenium, (Eds) Adam J. and Schwarz, K. P., Springer IAG, 125, 322-328, 2002a.

Švehla D. and Rothacher M.: CHAMP double-difference kinematic orbit with ambiguity resolution, First CHAMP Science Meeting, Potsdam, Germany, in: “ First CHAMP mission results for gravity, magnetic and atmospheric studies, (Eds) Reigber, Ch., Lühr, H., and Schwintzer, P., Springer-Verlag, pp. 70-77, 2003. 